\title{
OVER-EXPRESSION OF PEPTIDE DEFORMYLASE IN CHLOROPLASTS CONFERS ACTINONIN RESISTANCE, BUT IS NOT A SUITABLE SELECTIVE MARKER SYSTEM FOR PLASTID TRANSFORMATION
}

\author{
Alicia Fernández-San Millán, Patricia Obregón \& Jon Veramendi* \\ Instituto de Agrobiotecnología, Universidad Pública de Navarra-CSIC-Gobierno de Navarra, \\ Campus de Arrosadía, 31006-Pamplona, Spain
}

Keywords: deformylase, plastid transformation, tobacco, selective marker

*Author for correspondence. Tel: +34-948-168033. Fax: +34-948-232191

E-mail: jon@unavarra.es

\begin{abstract}
Arabidopsis thaliana peptide deformylase PDF1B was expressed in tobacco chloroplasts using spectinomycin as the selective agent. The foreign protein accumulated in chloroplasts $(6 \%$ of the total soluble protein) and was enzymatically active. Transplastomic plants were evaluated for resistance to the peptide deformylase inhibitor actinonin. In vitro seed germination in the presence of actinonin and in planta application of the inhibitor demonstrated the resistance of the transformed plants. In addition, transgenic leaf explants were able to develop shoots via organogenesis in the presence of actinonin. However, when the combination of the PDF1B gene and actinonin was used as the primary selective marker system for chloroplast transformation of tobacco, all developed shoots were escapes. Therefore, under the experimental conditions tested, the use of this system for plastid transformation would be limited to function as a secondary selective marker.
\end{abstract}




\section{Introduction}

The availability of suitable selective marker genes and selective agents is critical for the development of efficient plant transformation protocols. The most widely used selective system for plastid transformation is the aadA gene from $E$. coli that confers resistance to spectinomycin and streptomycin (Svab and Maliga, 1993). Spectinomycin selection is very effective and yields between 0.5 and 5 transplastomic lines per bombarded leaf in tobacco (Maliga, 2004). Kanamycin resistance, conferred by the bacterial nptII (Carrer et al., 1993) or aphA-6 (Huang et al., 2002) genes, was also appropriate for the recovery of plastid transformants, despite the lower overall transformation efficiency compared to that of the aadA gene. The tobacco feedback-insensitive anthranilate synthase $\alpha$-subunit, which catalyses the first reaction in the tryptophan biosynthetic pathway, has been successfully used for the recovery of tobacco transplastomic plants (Barone et al., 2009). The tryptophan analogue 7-methyl-DL-tryptophan was used as the selective agent. With this selection scheme, one resistant line was obtained out of 20 bombarded leaves. Recently, the chloramphenicol acetyl transferase gene cat has been reported as a new selectable marker for plastid transformation of tobacco (Li et al., 2010). Although selection with cat, in the presence of chloramphenicol, was less efficient than with $\operatorname{aad} A$, the cat marker offers the advantage of not producing spontaneous antibiotic-resistance mutants. In addition to these primary selective markers, there are also secondary markers that are unsuitable for direct selection when only a few plastid DNA copies are transformed but that are able to confer a selective advantage when most genome copies are transformed (Maliga, 2004). Examples are the bacterial bar gene that detoxifies the herbicide phosphinothricin (Lutz et al., 2001) and the CP4 gene that confers resistance to the herbicide glyphosate (Ye et al., 2003). Due to the limited repertoire of marker genes available for plastid transformation, it would be highly desirable to increase the range of selective marker systems. One of the major obstacles to extending this chloroplast-based technology to major important crops is the lack of suitable selective markers (Daniell et al., 2002). Some species are naturally resistant to antibiotics used in plastid transformation (e.g., maize and rice are spectinomycin-resistant) (Fromm et al., 1987). In other species such as cotton, transplastomic plants were not recovered after bombardment and spectinomycin selection, even at low concentrations. However, plastid transformation was achieved under kanamycin selection (Kumar et al., 2004). Therefore, a wider variety of selective marker systems would ease the transformation of recalcitrant species. Whereas protein synthesis begins with methionine in the cytosol, the process of translational initiation of proteins in plastids and mitochondria is similar to that of eubacteria, resulting in the incorporation of formyl-methionine at the $\mathrm{N}$-terminus. The formyl group is later excised from the nascent polypeptide by a peptide deformylase (PDF). Deformylation is an essential process both in prokaryotes and eukaryotes, despite proteomics analysis have also revealed a number of cases in which plastid-encoded proteins retain the formyl group (Giglione and Meinnel, 2001). PDF mutants in E. coli are lethal (Mazel et al., 1994; Meinnel and Blanquet, 1994), resulting in an albino phenotype in Arabidopsis thaliana (Giglione et al., 2003) and display chlorosis and 
growth retardation in rice (Moon et al., 2008). Moreover, excision of the formyl group is a necessary step for the subsequent removal of the initial methionine by a methionine aminopeptidase in proteins that do not retain this amino acid at the $\mathrm{N}$-terminus in their mature forms. These post-translational modifications contribute to the turnover of the formate and methionine compounds in the stroma of plastids.

Actinonin, a pseudo-peptide hydroxamate derivative, is a selective and potent inhibitor of bacterial deformylases (Chen et al., 2000). Plant deformylases are also very sensitive to actinonin (Giglione and Meinnel, 2001). The effects of actinonin treatment in plants include the inhibition of seed germination, an induced albino phenotype in germinated seedlings, chlorosis and a severe reduction in plant growth (Dirk et al., 2001; Giglione et al., 2003; Serero et al., 2001). The inhibition of peptide deformylase resulted in decreased D1 protein accumulation in tobacco chloroplasts, probably due to incorrect cotranslational processing, leading to photosystem II disassembly and leaf necrosis (Hou et al., 2004). Therefore, actinonin and other functional homologues have been proposed as a new class of broad-spectrum herbicides (Hou et al., 2006).

Two peptide deformylase genes have been identified in Arabidopsis thaliana (Dirk et al., 2001; Giglione et al., 2000) and three in rice (Moon et al., 2008). Arabidopsis deformylase genes have been extensively studied, and subcellular localization experiments show that AtPDF1A and AtPDF1B are present in mitochondria and chloroplasts (Hou et al., 2007; Moon et al., 2008). Due to the low similarity between AtPDF1B and the E. coli deformylase (Dirk et al., 2001), it is conceivable that a compound without antibacterial activity that specifically inhibits plant PDF1B could be developed. In this context, AtPDF1B would be an ideal selective marker for plant transformation that would eliminate the use of marker genes conferring antibiotic-resistance, avoiding the biosafety concerns about the use of such genes derived from micro-organisms (Miki and McHugh, 2004). With this objective, transgenic nuclear tobacco plants expressing different forms of Arabidopsis PDF were evaluated for resistance to actinonin (Hou et al., 2007). The over-expression of AtPDF1A.2 or AtPDF1B resulted in resistance to actinonin, suggesting that the combination of plant PDF and actinonin could serve as a native gene selective marker system for plant transformation vectors.

In the present work, we expressed the AtPDF1B gene in tobacco chloroplasts and analysed the resistance of the transplastomic plants to actinonin. The aim of this study was to evaluate the potential of plant deformylases as new selective marker genes for plastid transformation.

\section{Materials and methods}

\section{Construction of the chloroplast expression vector}

The peptide deformylase PDF1B cDNA (clone RAFL07-10-E08) from Arabidopsis thaliana was obtained from the Riken Arabidopsis Full-Length Clone Database (www.brc.riken.go.jp/lab/epd/Eng/). For cloning into the chloroplast expression pAF vector (Fernandez-San Millan et al. 2008), the PDF1B gene was amplified by PCR with the following 
primers: 5'-GGTACCGGAGGCAACCATGGCCGTCTGTAACTGCTTCC-3' and 5'CGAGCGgCCGCTTAATGgTGATGgTGATGgTGACGTTTGCCAAAACCAAC -3'. The PCR product, which included new $\mathrm{Ncol}$ and Notl restriction sites and the sequence corresponding to a $6 x$ His-tag in the carboxy terminal, was cloned into the pGEM-T easy vector (Promega). PDF1B (840 bp) was digested with Ncol/Notl and fused to the promoter and 5'-untranslated region of the tobacco chloroplast $p s b A$ gene in a pKS intermediate vector. Finally, the fusion was digested with EcoRVINotl and introduced into the pAF vector. The final pAF-PDF1B clone was sequenced, and its functionality was tested by western blot using $E$. coli soluble extracts.

\section{Chloroplast transformation by particle bombardment under spectinomycin selection}

Gold microprojectiles $(0.6 \mu \mathrm{m})$ coated with pAF-PDF1B plasmid DNA were bombarded into tobacco (Nicotiana tabacum var. Petite Havana) in vitro-grown leaves using the biolistic device PDS1000/He (Bio-Rad) as previously described (Molina et al., 2004). After bombardment, leaves were cut into small pieces and cultured in Magenta vessels (Sigma) in RMOP medium (Murashige and Skoog mineral salts, $30 \mathrm{~g} / \mathrm{L}$ sucrose, $0.1 \mathrm{mg} / \mathrm{L}$ NAA, $1 \mathrm{mg} / \mathrm{L}$ BAP, $1 \mathrm{mg} / \mathrm{L}$ thiamine, $100 \mathrm{mg} / \mathrm{L}$ myo-inositol and $6 \mathrm{~g} / \mathrm{L}$ phytagar, $\mathrm{pH} 5.8$ ) supplemented with $500 \mathrm{mg} / \mathrm{L}$ spectinomycin dihydrochloride as the selective agent. Resistant shoots were subjected to a second round of selection under the same conditions. Regenerated plants were transplanted and grown in a phytotron for further experiments.

\section{Southern blot analysis}

Total plant DNA was extracted from leaves of two-month-old plants using the cetyltrimethylammonium bromide (CTAB) procedure (Murray and Thompson, 1980). Twenty micrograms of total DNA was digested with HindllI, separated on a $0.8 \%(\mathrm{w} / \mathrm{v})$ agarose gel and transferred to a nylon membrane. The pUC-CT vector (Lee et al., 2003), digested with $B g / l l$ and $B a m H I$, generated a $0.81 \mathrm{~kb}$ probe $(\mathrm{P} 1)$ homologous to the flanking sequences. A $0.32 \mathrm{~kb}$ fragment (P2 probe) of the PDF1B gene was obtained by Smal-Notl digestion of the pAF-PDF1B vector. Hybridisation was performed using the chemiluminiscent AlkPhos direct labelling-detection system (GE Healthcare). After Southern blot confirmation, plants were transferred to soil. Seeds from the $T_{0}$ generation were in vitro germinated on spectinomycin selection medium. The $T_{1}$ seedlings were isolated and cultured for four weeks in Magenta vessels. Finally, plants were transferred to pots.

\section{Purification of recombinant PDF1B from transplastomic plants}

Soluble PDF1B was affinity-purified using Ni-NTA Agarose (Qiagen) according to the manufacturer's protocol and buffers. Briefly, $5 \mathrm{~g}$ of leaves was ground in liquid nitrogen and mixed with sonication buffer $\left(50 \mathrm{mM} \mathrm{NaH} \mathrm{PO}_{4}, 300 \mathrm{mM} \mathrm{NaCl}\right.$ and $10 \mathrm{mM}$ Imidazole, $\mathrm{pH}$ 8). After $5 \mathrm{~min}$ of sonication and $15 \mathrm{~min}$ of centrifugation at $20,000 \mathrm{~g}$, the supernatants were filtered through two layers of miracloth. Filtrates were mixed with $500 \mu \mathrm{L}$ of Ni-NTA agarose, incubated in a rotary shaker for $90 \mathrm{~min}$ at $4^{\circ} \mathrm{C}$ and passed through a gravity-flow chromatography column 
to separate the agarose with affinity-attached proteins from the debris. The column was washed with $20 \mathrm{~mL}$ of washing buffer (50 mM NaH $\mathrm{PO}_{4}, 300 \mathrm{mM} \mathrm{NaCl}$ and $20 \mathrm{mM}$ Imidazole, $\mathrm{pH}$ 8), and proteins were eluted in $8 \mathrm{~mL}$ of elution buffer $\left(50 \mathrm{mM} \mathrm{NaH}_{2} \mathrm{PO}_{4}, 300 \mathrm{mM} \mathrm{NaCl}\right.$ and $250 \mathrm{mM}$ Imidazole, $\mathrm{pH}$ 8). The eluates were concentrated and dialysed against $50 \mathrm{mM}$ Hepes and 10 $\mathrm{mM} \mathrm{CINa}$ at $\mathrm{pH} 7$ in a Vivaspin 10 MWCO PES concentrator (Sartorius). Proteins were quantified by a Bradford assay (Bio-Rad). Purified samples were stored at $4^{\circ} \mathrm{C}$ until the activity assays were performed.

\section{Western blot analysis and PDF quantification}

Leaf discs (100 $\mathrm{mg}$ ) were ground in liquid nitrogen and homogenised in phosphate-buffered saline (PBS) at $\mathrm{pH} 7.4$. After centrifugation at $20,000 \mathrm{~g}$ for $5 \mathrm{~min}$, the supernatant was the soluble fraction. The pellet was resuspended in $200 \mu \mathrm{L}$ of loading buffer $(250 \mathrm{mM}$ Tris- $\mathrm{HCl}, \mathrm{pH}$ $6.8,4 \%$ SDS, $10 \%$ glycerol, $10 \% \beta$-mercaptoethanol and $0.01 \%$ bromophenol blue) and boiled for $5 \mathrm{~min}$. After centrifugation at $20,000 \mathrm{~g}$ for $5 \mathrm{~min}$, the supernatant was considered the insoluble fraction. For $E$. coli expressing PDF1B from the pAF-PDF1B vector, $3 \mathrm{~mL}$ of an overnight culture was pelleted, resuspended in $100 \mu \mathrm{L}$ of loading buffer and boiled for 5 min.

Proteins were separated on $12 \%$ SDS (w/v) polyacrylamide gels and transferred onto nitrocellulose membranes (Hybond C, GE Healthcare), blocked overnight in PBS with $0.1 \%(\mathrm{v} / \mathrm{v})$ Tween-20 (PBS-T) and 5\% skim milk (PBS-TM) and incubated for $1 \mathrm{~h}$ with HRP-conjugated anti-His antibody (Sigma) diluted 1:20 000 in PBS-TM. After two washes of $10 \mathrm{~min}$ in PBS-T, the specific signal was detected using the Advanced ECL system (GE Healthcare) according to the manufacturer's instructions. Affinity-purified PDF1B, as described previously, was used as the standard in the range of 100-600 ng. Transgenic leaf extracts were diluted to fit within the linear range of the standards and measured by densitometry with the GeneTools analyser (Syngene).

\section{Peptide deformylase fluorescence assay}

The deformylase activity was measured in a fluorescent assay using fluorescamine to monitor the release of formate from a $\mathrm{N}$-formylated peptide substrate and to detect the formation of the new amino group (Haas et al., 2001). Fluorescamine reacts directly with primary amines to yield highly fluorescent fluorophors. The substrate for deformylation was formyl-Met-Ala-Ser (fMAS, Bachem, Germany). The deformylase activity was measured in 96-well microtitre plates. Different dilutions (150-1200 nM) of the affinity-purified enzyme and variable concentrations (0$10 \mathrm{mM}$ ) of the peptide fMAS were incubated for $40 \mathrm{~min}$ at room temperature in a $50 \mu \mathrm{L}$ reaction volume (50 mM HEPES and $10 \mathrm{mM} \mathrm{NaCl}, \mathrm{pH}$ 7.3). After the addition of $20 \mu \mathrm{L}$ of a fluorescamine (Sigma) solution $(0.2 \mu \mathrm{g} / \mathrm{mL}$ in $100 \% \mathrm{DMSO})$, the fluorescence was determined using a fluorescence plate reader (Multiskan Ex, Labsystems) with the excitation wavelength at $390 \mathrm{~nm}$ and the emission wavelength at $485 \mathrm{~nm}$. The results were expressed as relative fluorescence units (RFU). 


\section{Antibiotic susceptibility tests}

Actinonin inhibitory effect on deformylation activity

Affinity-purified PDF1B (1200 nM) from transplastomic tobacco plants was pre-incubated for 15 min with $0-1000 \mathrm{nM}$ actinonin prior to initiating the reaction by adding $7.5 \mathrm{mM}$ fMAS. After a 40 min incubation at room temperature, $20 \mu \mathrm{L}$ of fluorescamine solution was added, and the fluorescence was measured as explained above.

Germination of tobacco seeds with actinonin

Tobacco seeds from two transplastomic lines were germinated in the wells of 96-well microtitre plates containing Murashige and Skoog mineral salts including vitamins (Duchefa), $30 \mathrm{~g} / \mathrm{L}$ sucrose, $\mathrm{pH} 5.8$, and $0.6 \%$ microagar (Duchefa) in the absence or presence of different levels of actinonin (300-600-1200 $\mu \mathrm{M})$. As control, seeds were also germinated on medium containing $500 \mathrm{mg} / \mathrm{L}$ spectinomycin. Plates were incubated for 30 days in the culture chamber, and seedling development was recorded.

Actinonin treatment of leaves

Mature leaves of transgenic and wild-type plants grown in a phytotron were treated daily with 5 $\mu \mathrm{L}$ of $0.1,5$ or $10 \mathrm{mM}$ actinonin in $0.05 \%$ ( $\mathrm{v} / \mathrm{v}$ ) Tween-20 for six days. Drops were placed every day with a pipette tip on the same point of the adaxial side of the leaves. Three replicates on two different plants were performed.

Organogenesis of transplastomic leaves in actinonin-containing media

Leaves from phytotron-grown $\mathrm{T} 1$ transplastomic plants were cut into $5 \times 5 \mathrm{~mm}$ pieces and placed abaxial side down onto RMOP medium supplemented or not with actinonin (600-1200 $\mu \mathrm{M})$ or $500 \mathrm{mg} / \mathrm{L}$ of spectinomycin as the control. Twenty-four-well plates were used with $700 \mu \mathrm{L}$ of medium and one explant per well.

\section{Bombardment and regeneration of chloroplast transgenic plants under actinonin selection}

Bombardment with pAF-PDF1B vector was performed as described above. After bombardment, leaves were cut into small pieces (approximately 30 explants per leaf) and cultured in six-well plates containing three explants and $6 \mathrm{~mL}$ of RMOP medium supplemented with $0,300,600$, 800,1000 or $1200 \mu \mathrm{M}$ actinonin per well. As the control, RMOP, with or without spectinomycin dihydrochloride (500 mg/L) as the selection agent, was used. Explants were transferred to fresh medium after three to four weeks. Shoots $(0.5-1 \mathrm{~cm}$ in length) developed after seven weeks of culture were cut and subcultured in rooting medium (Murashige and Skoog mineral salts including vitamins, $30 \mathrm{~g} / \mathrm{L}$ sucrose, $\mathrm{pH} 5.8$, and $0.6 \%$ microagar) supplemented with $500 \mathrm{mg} / \mathrm{L}$ spectinomycin to evaluate the transgenic nature of the regenerants. Total DNA from shoots developed under 600-1200 $\mu \mathrm{M}$ actinonin was extracted and subjected to a PCR analysis to detect the insertion of $A t P D F 1 B$ and aadA genes in the plastid genome. Control shoots developed in the presence of spectinomycin were analysed by $P C R$ with primers $3 P$ and $3 M$ (Figure 1a) to detect the insertion of the aadA gene. 


\section{Results}

\section{Vector construction and generation of transplastomic plants}

The pAF vector (Fernandez-San Millan et al., 2008) was used for tobacco chloroplast transformation. This vector includes the $\operatorname{trn} l$ and $\operatorname{trn} A$ border sequences, homologous to the inverted repeat regions of the tobacco plastid genome, and the aminoglycoside 3'adenylyltransferase $(\operatorname{aad} A)$ gene from $E$. coli, which confers resistance to spectinomycin. Border sequences mediate the integration of the foreign DNA into the plastid genome by homologous recombination. The Arabidopsis thaliana PDF1B cDNA was inserted in the pAF vector under the control of the promoter and 5'-untranslated region of the $p s b A$ gene, yielding the final PAF-PDF1B vector (Figure 1a). The PDF1B recombinant protein included the original transit peptide sequence and a carboxy terminal 6xHis-tag. Previously, it was shown that a Cterminal His-tag fusion did not affect PDF enzymatic activity (Dirk et al., 2001). We selected $P D F 1 B$ instead of PDF1A because of the higher actinonin tolerance of PDF1A in in vitro assays (Dirk et al., 2001) and in nuclear transgenic tobacco plants over-expressing either PDF1B or PDF1A (Hou et al., 2007).

Chloroplast transformation was performed by particle bombardment following previously described protocols (Molina et al., 2004). Bombarded leaf fragments were cultured under the selection of spectinomycin to obtain transformed shoots.

\section{Determination of chloroplast integration and homoplasmy}

Plantlets that developed after the transformation process were analysed for transgene integration in the plastid genome by PCR with primers $3 \mathrm{P}$ and $3 \mathrm{M}$. Primer $3 \mathrm{P}$ binds on the plastid genome upstream of the $\mathrm{trnl}$ gene outside the vector integration site, and primer $3 \mathrm{M}$ binds to the aadA gene (Figure 1a). The resulting PCR product $(1.65 \mathrm{~kb})$ is specific for transformed plastid genomes and cannot be amplified from nuclear transgenic plants. Ten independent plantlets were analysed, and all of them were PCR-positive, whereas wild-type samples did not amplify any fragment (data not shown). Subsequently, a Southern blot analysis of five plants was performed to confirm site-specific integration and to verify homoplasmy. Total plant DNA was digested with HindIII. DNA from non-transformed plants produced a fragment of $7.8 \mathrm{~kb}$ when it was hybridised with the flanking-region probe (P1, Figure 1b,c). Transformed plants produced a fragment of $10.1 \mathrm{~kb}$. All five independent plants analysed were homoplasmic, showing the fragment of $10.1 \mathrm{~kb}$ but lacking the signal of $7.8 \mathrm{~kb}$ corresponding to the wild-type plastid genome (Figure 1c). To confirm that the $10.1 \mathrm{~kb}$ fragment contained the PDF1B sequence, the same blot was re-probed with the P2 probe (Figure 1b). As expected, hybridisation was observed in the five transgenic lines but was absent in the non-transformed plant (Figure 1d). Seed germination and green plantlet development on spectinomycincontaining medium confirmed that homoplasmy was maintained in the $T_{1}$ generation of both transplastomic lines (data not shown). 


\section{Expression and quantification of the PDF1B protein}

The Arabidopsis thaliana PDF1B protein expressed in transgenic chloroplasts was detected by western blot with an anti-histidine antibody. A 25-kDa band of the expected size for the mature PDF1B was detected (Figure 2a)(Dirk et al., 2001). The recombinant protein included its original $\mathrm{N}$-terminal transit peptide sequence of 56 residues that presumably would be cleaved in the chloroplast by a general stromal processing peptidase after the synthesis of the polypeptide. To confirm this point, protein extracts from $E$. coli transformed with pAF-PDF1B were subjected to western blot. As expected, a band of higher molecular mass was detected (Figure 2a), denoting non-processing of the signal peptide in E. coli (Dirk et al., 2001) and confirming its removal when PDF1B was synthesised in the chloroplast. The enzymatic machinery of the plastid is able to process transit peptides of recombinant proteins expressed in the chloroplast. For example, cleavage of the transit peptide from the Rubisco small subunit fused to the human serum albumin expressed in tobacco chloroplasts was previously demonstrated (Fernandez-San Millan et al., 2007).

The foreign protein was mainly detected in the soluble fraction as a single band (Figure $2 b$ ), suggesting the absence of insoluble aggregates of multimeric structures, frequently present when high expression levels are achieved in the chloroplast (Fernández-San Millán et al., 2003; Fernandez-San Millan et al., 2008). This point was confirmed when protein samples were run under native conditions, and only the monomeric band was detected (data not shown). The effect of leaf age on PDF1B protein expression was analysed in plants grown in the greenhouse for 60 days. Young, mature and old leaves (12, 8 and 3 leaves, respectively, counted from the bottom of the plant) were selected. Strong bands were observed in young and mature leaves, but the signal was very weak in old leaves (Figure $2 b$ ). Lack of accumulation of PDF1B in old leaves could indicate a high sensitivity of this protein to chloroplast proteases, though no degradation products were detected in the western blot. As previously shown, unstable foreign proteins expressed in chloroplasts show a decline in protein accumulation with leaf age (Farran et al., 2008).

The amount of PDF1B accumulated in the chloroplast was quantified by western blot using affinity-purified PDF1B as the standard. Different amounts of protein extracts were loaded onto the gel. After developing the film, the blot was analysed by densitometry (Figure 2c). The mean value of three independent experiments was calculated, and the expression level of the foreign protein was estimated as $0.61 \mathrm{mg} / \mathrm{g}$ fresh weight of leaf (equivalent to $6 \%$ of the total soluble protein). To confirm this data, total protein extracts from transplastomic and wild-type plants were separated by SDS-PAGE and stained with Coomassie brilliant blue. A 25-kDa band corresponding to the PDF1B protein was visualised in the transplastomic plant but was absent in the non-transformed plant (Figure 2d). Transplastomic plants showed some phenotypic differences such as the light green colour of leaves in comparison to wild-type plants (data not shown). Additionally, lower Rubisco levels were detected in these plants (Figure 2d). However, plants developed normally and set seeds. 


\section{Chloroplast-produced PDF1B catalyses peptide deformylation}

The affinity-purified recombinant PDF1B catalysed the deformylation of the fMAS peptide, a common substrate used in enzymatic deformylase assays (Haas et al., 2001). As expected, fluorescence increased with higher concentrations of the peptide substrate (Figure 3a). In addition, an increase in relative fluorescence units was detected with increasing levels of deformylase.

Actinonin is a naturally occurring inhibitor of bacterial peptide deformylase. It was previously shown that actinonin inhibited the enzyme activity of Arabidopsis thaliana PDF1B (Dirk et al., 2001). We found that actinonin also inhibited the chloroplast-produced PDF1B (Figure 3b). Over $80 \%$ inhibition of the enzymatic activity was observed with $10 \mathrm{nM}$ actinonin.

\section{Over-expression of PDF1B in the chloroplast confers resistance to the peptide deformylase inhibitor actinonin}

Seeds from two independent transplastomic lines were in vitro germinated in the presence of different concentrations of actinonin. Wild-type seedlings showed small and bleached cotyledons and growth arrest even at the lowest level of actinonin, similar to the effect observed with spectinomycin (Figure 4a). In contrast, transplastomic plantlets developed green cotyledons and true leaves. A slight growth delay was observed in plant development, especially at the highest concentration of actinonin $(1200 \mu \mathrm{M})$. Transplastomic PDF1B plants were transplanted, displaying a normal fertility. In planta experiments also demonstrated that transplastomic plants were actinonin-resistant. Six days after leaf treatment with actinonin droplets, bleached spots were observed in wild-type leaves, but transformed leaves were unaffected irrespective of the actinonin concentration (Figure 4c). The sensitivity of tobacco plants to actinonin was previously demonstrated in vitro (Hou et al., 2007) and in vivo (Hou et al., 2004).

To assess the organogenic capacity of the transformed plants, leaf explants were cultured on RMOP medium supplemented with actinonin. Thirty days after culture with $600 \mu \mathrm{M}$ of actinonin, leaf explants expanded and developed buds in a similar way to the control without actinonin or the medium including spectinomycin (Figure 4b). Buds were also produced with $1200 \mu \mathrm{M}$ of actinonin, though the explant growth was reduced. Organogenesis on non-transformed leaf explants was totally inhibited by $1200 \mu \mathrm{M}$ of actinonin. Under these conditions, growth was also arrested, and explants bleached and died (Figure 4b). At $600 \mu \mathrm{M}$ of actinonin, despite a growth delay, some buds developed. These results suggest that actinonin should be used as selective agent at concentrations higher than $600 \mu \mathrm{M}$ to completely avoid the development of nontransformed shoots.

\section{PDF1B is not an appropriate selective marker for chloroplast transformation}

After confirming that AtPDF1B could be expressed in the stroma of the chloroplast, was enzymatically active and conferred resistance to actinonin, we wanted to test whether the 
combination of the PDF1B gene with a PDF inhibitor could serve as a selective marker system for chloroplast transformation. The pAF-PDF1B vector, described above, was used for particle bombardment of in vitro-grown leaves. Bombarded leaf explants were grown under different concentrations of actinonin, instead of the antibiotic spectinomycin that was used in the first transformation experiment. Here, spectinomycin was employed as the control selective marker. Fifty days after bombardment, green shoots had developed under all levels of actinonin treatment, with the exception of $1200 \mu \mathrm{M}$, where the initial explants turned brown (Figure 5a). The 29 shoots developed under the control of spectinomycin selection were analysed at the molecular level. PCR analysis with primers that bind on the trnl and aadA genes (Molina et al., 2004) confirmed that $100 \%$ of the shoots were transformants rather than spontaneous spectinomycin resistant mutants (data not shown). All shoots (182) developed in the presence of actinonin were excised and transferred to a rooting medium including spectinomycin. If the transgenes had inserted in the plastid genome, developed shoots should be spectinomycinresistant. However, all shoots bleached, suggesting that they were escapes developed in the presence of actinonin. Another explanation could be that, under low actinonin selective pressure, few copies of the transformed plastid genome were present relative to the wild-type plastid genome, facilitating the development of non-transformed spectinomycin-sensitive shoots. To analyse the presence of the transgenes, total DNA from shoots developed under 600-1200 $\mu \mathrm{M}$ actinonin treatment was extracted and subjected to a PCR analysis with primers specific for AtPDF1B and aadA. No amplification of fragments of the expected sizes was observed (data not shown). A new experiment with an increased number of bombardments (12/concentration of actinonin) was performed and selection carried out through the higher levels analysed (800, 1000 and $1200 \mu \mathrm{M})$ (Figure 5c). Again, organogenesis was not observed in the highest concentration of actinonin, and all shoots (118) developed in the media containing 800 or $1000 \mu \mathrm{M}$ of the PDF inhibitor bleached when transferred to a rooting medium including spectinomycin. PCR analysis of DNA from these shoots to detect the presence of AtPDF1B and aadA were also negative (data not shown). The 23 shoots developed under the control spectinomycin selection were analysed by PCR and 22 of them resulted to be true transformants and one was a spontaneous spectinomycin resistant mutant (data not shown)

\section{Discussion}

Actinonin has a phytotoxic effect in crop plants (Giglione et al., 2003; Moon et al., 2008; Serero et al., 2001) and in weed species (Hou et al., 2006). This compound specifically inhibits PDF, a metallopeptidase that catalyses the removal of the formyl group from formyl-methionine, the initial amino acid for all proteins synthesised in the plastids. Over-expression of PDF1B by nuclear transformation conferred protection to actinonin, suggesting the potential use of this system as a new selective marker (Hou et al., 2007). To test this hypothesis, transgenic tobacco plants expressing the Arabidopsis thaliana PDF1B in their chloroplasts were developed in this study, and high expression levels of the foreign protein were achieved. The recombinant protein 
was also visualised by PAGE and Coomassie brilliant blue staining. The over-expression of the PDF1B protein in the stroma of the chloroplast made the transplastomic plants resistant to actinonin. However, in our hands, the combination of AtPDF1B and actinonin as the primary selective marker system did not allow the recovery of transplastomic tobacco plants. Therefore, the use of this system would be limited to function as secondary selective marker, equivalent to bar and CP4 genes (Lutz et al., 2001; Ye et al., 2003).

In Arabidopsis wild-type plants, PDF1B is expressed at very low levels in the stroma of the chloroplast (Dirk et al., 2001). In our transplastomic plants, we presumably have much higher levels of deformylase than in wild-type plants and approximately 100-fold higher expression than in nuclear transgenic tobacco plants transformed with the same gene (Hou et al., 2007). As a consequence, very high levels of deformylase activity were expected. Notwithstanding this, no plastid transformants were recovered when actinonin and AtPDF1B were used as the primary selective system. Therefore, where does the inefficiency of this marker system lie? Several hypotheses can be formed. Firstly, the Arabidopsis PDF1B protein produced in the tobacco chloroplast is not properly assembled. Analysis of the crystal structure and gel filtration chromatography suggests that AtPDF1B exists as a dimer (Dirk et al., 2008). Our results showed that heterologous PDF1B is present in tobacco chloroplasts as a monomer, and no dimer was detected (Figure 2a,b). The dimer conformation could favour protein stability and increased enzymatic activity. Secondly, tobacco has been reported to be more resistant to actinonin compared to other species such as pea and Arabidopsis (Hou et al., 2004), and this could favour the development of escapes. Organogenesis in wild-type explants was completely inhibited at the highest concentration of actinonin $(1200 \mu \mathrm{M})$ but not at lower levels (Figure 4b), where shoots developed. Under these circumstances, it would be normal in a transformation experiment to observe the development of non-transformed plants under low selective pressure. Thirdly, because the in vitro transformation process lasts several weeks, it is conceivable that selective pressure decreases with time, allowing the formation of buds from non-transformed cells. It has been demonstrated that actinonin is rapidly metabolised and inactivated after absorption, with only $17 \%$ of the original compound remaining $48 \mathrm{~h}$ after in vivo application (Hou et al., 2006). However, the observed results indicate that some active actinonin was present during the whole period of in vitro culture. Lower numbers of shoots and growth delay were noticed compared to actinonin-free medium at the end of the culture period (Figure 5), suggesting that actinonin stability is not a key issue in this study.

In conclusion, though Arabidopsis PDF1B can be expressed at high levels in tobacco chloroplasts without deleterious effects on plant growth and development, and the recombinant protein conserves its enzymatic activity, the combination of actinonin and PDF does not yield a suitable primary selective marker system for plastid transformation.

\section{Acknowledgements}


This work was partially supported by Grant BIO2005-00155 from the Ministerio de Educación y Ciencia (Spain).

\section{References}

Barone P, Zhang XH and Widholm JM (2009) Tobacco plastid transformation using the feedback-insensitive anthranilate synthase [alpha]-subunit of tobacco (ASA2) as a new selectable marker. J Exp Bot 60:3195-3202.

Carrer H, Hockenberry TN, Svab Z and Maliga P (1993) Kanamycin resistance as a selectable marker for plastid transformation in tobacco. Mol Gen Genet 241:49-56.

Chen DZ, Patel DV, Hackbarth CJ, Wang W, Dreyer G, Young DC, Margolis PS, Wu C, Ni ZJ, Trias J, White RJ and Yuan Z (2000) Actinonin, a naturally occurring antibacterial agent, is a potent deformylase inhibitor. Biochemistry 39:1256-1262.

Daniell H, Khan MS and Allison L (2002) Milestones in chloroplast genetic engineering: an environmentally friendly era in biotechnology. Trends Plant Sci 7:84-91.

Dirk LM, Schmidt JJ, Cai Y, Barnes JC, Hanger KM, Nayak NR, Williams MA, Grossman RB, Houtz RL and Rodgers DW (2008) Insights into the substrate specificity of plant peptide deformylase, an essential enzyme with potential for the development of novel biotechnology applications in agriculture. Biochem J 413:417-427.

Dirk LM, Williams MA and Houtz RL (2001) Eukaryotic peptide deformylases. Nuclear-encoded and chloroplast-targeted enzymes in Arabidopsis. Plant Physiol 127:97-107.

Farran I, Rio-Manterola F, Iniguez M, Garate S, Prieto J and Mingo-Castel AM (2008) Highdensity seedling expression system for the production of bioactive human cardiotrophin1 , a potential therapeutic cytokine, in transgenic tobacco chloroplasts. Plant Biotech $\mathrm{J}$ 6:516-527.

Fernandez-San Millan A, Farran I, Molina A, Mingo-Castel AM and Veramendi J (2007) Expression of recombinant proteins lacking methionine as $\mathrm{N}$-terminal amino acid in plastids: human serum albumin as a case study. J Biotechnol 127:593-604.

Fernández-San Millán A, Mingo-Castel AM, Miller M and Daniell H (2003) A chloroplast transgenic approach to hyper-express and purify Human Serum Albumin, a protein susceptible to proteolytic degradation. Plant Biotech J 1:71-79.

Fernandez-San Millan A, Ortigosa SM, Hervas-Stubbs S, Corral-Martinez P, Segui-Simarro JM, Gaetan J, Coursaget P and Veramendi J (2008) Human papillomavirus L1 protein expressed in tobacco chloroplasts self-assembles into virus-like particles that are highly immunogenic. Plant Biotech J 6:427-441.

Fromm H, Edelman M, Aviv D and Galun E (1987) The molecular basis for rRNA-dependent spectinomycin resistance in Nicotiana chloroplasts. EMBO J 6:3233-3237.

Giglione C and Meinnel T (2001) Organellar peptide deformylases: universality of the N-terminal methionine cleavage mechanism. Trends Plant Sci 6:566-572.

Giglione C, Serero A, Pierre M, Boisson B and Meinnel T (2000) Identification of eukaryotic peptide deformylases reveals universality of $\mathrm{N}$-terminal protein processing mechanisms. EMBO J 19:5916-5929.

Giglione C, Vallon O and Meinnel T (2003) Control of protein life-span by N-terminal methionine excision. EMBO J 22:13-23.

Haas M, Beyer D, Gahlmann R and Freiberg C (2001) YkrB is the main peptide deformylase in Bacillus subtilis, a eubacterium containing two functional peptide deformylases. Microbiology 147:1783-1791.

Hou C-X, Dirk LMA, Goodman JP and Williams MA (2006) Metabolism of the peptide deformylase inhibitor actinonin in tobacco. Weed Science 54:246-254.

Hou C-X, Dirk LMA and Williams MA (2004) Inhibition of peptide deformylase in Nicotiana tabacum leads to decreased D1 protein accumulation, ultimately resulting in a reduction of photosystem II complexes. American Journal of Botany 91:1304-1311.

Hou CX, Dirk LM, Pattanaik S, Das NC, Maiti IB, Houtz RL and Williams MA (2007) Plant peptide deformylase: a novel selectable marker and herbicide target based on essential cotranslational chloroplast protein processing. Plant Biotechnol J 5:275-281.

Huang FC, Klaus SM, Herz S, Zou Z, Koop HU and Golds TJ (2002) Efficient plastid transformation in tobacco using the aphA-6 gene and kanamycin selection. Mol Genet Genomics 268:19-27. 
Kumar S, Dhingra A and Daniell H (2004) Stable transformation of the cotton plastid genome and maternal inheritance of transgenes. Plant Mol Biol 56:203-216.

Lee SB, Kwon HB, Kwon SJ, Park SC, Jeong MJ, Han SE, Byun MO and Daniell H (2003) Accumulation of trehalose within transgenic chloroplasts confers drought tolerance. Mol Breeding 11:1-13.

Li W, Ruf S and Bock R (2010) Chloramphenicol acetyltransferase as selectable marker for plastid transformation. Plant Mol Biol:DOI 10.1007/s11103-11010-19678-11104.

Lutz KA, Knapp JE and Maliga P (2001) Expression of bar in the plastid genome confers herbicide resistance. Plant Physiol 125:1585-1590.

Maliga P (2004) Plastid transformation in higher plants. Annu Rev Plant Biol 55:289-313.

Mazel D, Pochet S and Marliere P (1994) Genetic characterization of polypeptide deformylase, a distinctive enzyme of eubacterial translation. EMBO J 13:914-923.

Meinnel T and Blanquet S (1994) Characterization of the Thermus thermophilus locus encoding peptide deformylase and methionyl-tRNA(fMet) formyltransferase. J Bacteriol 176:73877390.

Miki B and McHugh S (2004) Selectable marker genes in transgenic plants: applications, alternatives and biosafety. J Biotechnol 107:193-232.

Molina A, Hervas-Stubbs S, Daniell H, Mingo-Castel AM and Veramendi J (2004) High-yield expression of a viral peptide animal vaccine in transgenic tobacco chloroplasts. Plant Biotech J 2:141-153.

Moon S, Giglione C, Lee DY, An S, Jeong DH, Meinnel T and An G (2008) Rice peptide deformylase PDF1B is crucial for development of chloroplasts. Plant Cell Physiol 49:1536-1546.

Murray SL and Thompson WF (1980) Rapid isolation of high molecular weight plant DNA. Nucleic Acids Res 8:4321-4326.

Serero A, Giglione C and Meinnel T (2001) Distinctive features of the two classes of eukaryotic peptide deformylases. J Mol Biol 314:695-708.

Svab Z and Maliga P (1993) High-frequency plastid transformation in tobacco by selection for a chimeric aadA gene. Proc Natl Acad Sci U S A 90:913-917.

Ye GN, Colburn SM, Xu CW, Hajdukiewicz PT and Staub JM (2003) Persistence of unselected transgenic DNA during a plastid transformation and segregation approach to herbicide resistance. Plant Physiol 133:402-410.

\section{Figure legends}

Figure 1. Schematic representation of plastid genome transformation with the Arabidopsis thaliana PDF1B gene (constructs not drawn to scale). (a) Map of the wild-type and PDF1Btransformed genomes. Regions for homologous recombination are underlined in the native chloroplast genome. The PDF1B sequence is driven by the $p s b A$ promoter. The annealing sites of the primers $(3 \mathrm{P}$ and $3 \mathrm{M})$ used to test integration of the cassette into the chloroplast genome are shown. (b) The 0.8-kb fragment (P1) of the targeting region for homologous recombination and the 0.32-kb PDF1B sequence (P2) were used as probes for a Southern blot analysis. (c, d) The Southern blot analysis of five independent transgenic lines (1-5) is shown. The same blot was probed with P1 (c) and P2 (d). 16S, 23S, trnl, trnA: original sequences of the chloroplast genome; aadA: aminoglycoside 3'-adenylyltransferase; Prrn: 16S rRNA promoter; PpsbA: psbA promoter and 5'-untranslated region; 3'psbA: terminator region of the $p s b A$ gene; WT: wild-type; M: molecular weight marker.

Figure 2. Characterization of PDF1B expression in transgenic chloroplasts. (a) Immunoblot analysis of transit peptide processing in tobacco chloroplasts. Extracts from E. coli transformants and from E. coli transformed with the empty vector $(C)$ were loaded as controls. (b) Soluble and insoluble proteins extracted from $1.5 \mathrm{mg}$ of leaf fresh weight per well were 
loaded on a $12 \%$ SDS-PAGE. Young $(\mathrm{Y})$, mature $(\mathrm{M})$ and old $(\mathrm{O})$ leaves were analysed. Total protein extract from wild-type (WT) leaf was loaded as the control. Blots were detected using anti-His HRP conjugated antibody. (c) PDF1B quantification in transgenic tobacco chloroplasts by western blot analysis. Total protein extracts from $1.2,0.6$ and $0.3 \mathrm{mg}$ of leaves were loaded for quantification. As standards, 100-600 ng of purified PDF1B protein were used. (d) Coomassie-blue stained SDS-PAGE gel. Fifteen micrograms of total protein extracted from transplastomic (T) or wild-type (WT) plants was loaded.

Figure 3. Activity of chloroplast-expressed PDF1B. (a) Effects of increasing concentrations of fMAS peptide addition on variable amounts of purified PDF1B: $150 \mathrm{nM}, 300 \mathrm{nM}, 600 \mathrm{nM}$ and 1200 nM. Control: wild-type protein extract purified in parallel to PDF1B. RFU: Relative fluorescence units. (b) Dose-response relationship of actinonin inhibition against PDF1B. The deformylation activity of $1200 \mathrm{nM}$ PDF1B was measured in the presence of $7.5 \mathrm{mM}$ fMAS and increasing concentrations of actinonin. The error bars represent the standard errors of the data from three independent experiments.

Figure 4. Tolerance of transplastomic plants to the peptide deformylase inhibitor actinonin. (a) Seeds from two transplastomic lines (T1 and T2) and wild-type plants (WT) were germinated on medium containing $0,300,600$ or $1200 \mathrm{mM}$ actinonin or $500 \mathrm{mg} / \mathrm{l}$ of spectinomycin. Seedlings were grown for 30 days. (b) Organogenesis of leaf explants from transplastomic $(T)$ or wild-type (WT) plants on RMOP medium supplemented with 0,600 or $1200 \mathrm{mM}$ of actinonin after 30 days. Spectinomycin was used as the positive control. (c) Effect of actinonin (six-day treatment with $5 \mathrm{ml}$ of solution) in leaves of transplastomic (T) or wild-type (WT) plants grown in a phytotron (bar represents $1 \mathrm{~cm}$ ).

Figure 5. Chloroplast transformation experiment with actinonin as the selective marker. (a) Shoot regeneration on leaf explants cultured on RMOP medium containing $0,300,600,800$, 1000 or $1200 \mathrm{mM}$ actinonin, or $500 \mathrm{mg} / \mathrm{l}$ of spectinomycin as the control, 50 days after bombardment with the pAF-PDF1B vector (bar represents $1 \mathrm{~cm}$ ). (b, c) Number of bombarded leaves, selected leaf pieces, regenerated shoots and resistant plantlets after transferring the shoots to a rooting media supplemented with $500 \mathrm{mg} / \mathrm{L}$ spectinomycin. Spec: spectinomycin. 